\title{
EL DIFÍCIL TRÁNSITO HACIA LA SEMIPRESENCIALIDAD EN LA EDUCACIÓN SUPERIOR: UN EJEMPLO IBEROAMERICANO
}

\author{
The difficult transition to blended learning in higher education: a Latin American \\ example
}

Elba Rosa Gómez Barajas

José Manuel Ríos Ariza

David Guillermo Pasillas Banda

Fecha de recepción: 08/07/2016

Fecha de aceptación: 09/11/2016

RESUMEN: En el Centro Universitario de los Valles (CUValles) de la Universidad de Guadalajara (México) se ha implementado un modelo semipresencial, en respuesta a algunas características de la población y de la zona donde se ubica. La investigación es parte de una evaluación realizada con estudiantes y profesores del Centro sobre el modelo. El tratamiento de los datos que se presentan son de tendencia central (media) y distribución porcentual. Los temas de interés que se abordan se relacionan son la aceptación que tiene el modelo, los motivos o razones por el que lo aceptan y la interactividad que tiene el profesorado con los estudiantes. Los datos reflejan que las principales causas o motivos por el que los estudiantes aceptan la semipresencialidad son personales, y reflejan una crítica en la implementación, ya que la mayoría creen que las actividades en línea no sustituyen la presencialidad. Por otro lado, los profesores se quejan de falta de formación para trabajar en un modelo semipresencial y del poco nivel de los estudiantes. La semipresencialidad, es una alternativa viable, para que las universidades presenciales iberoamericanas puedan aumentar su oferta educativa. Pero este tránsito no está exento de riesgos que pueden poner en peligro los posibles beneficios.

PALABRAS CLAVE: educación semipresencial, Educación Superior, demanda educativa, América Latina.

ABSTRACT: At Community College "de los Valles" (CuValles), institution from University of Guadalajara (México) a blended e-learning model has been developed. It was in order to satisfy the educational needs of local and regional population. This study, as part of a bigger research, has the aim of evaluating the blended model learning from $\mathrm{CuValles}$. The main analyzed dimensions were the acceptance of model, and the acceptance or not acceptances motives and reasons. Sample included students and lecturers from CuValles, and a descriptive analysis was conducted. The result was that the main acceptance reasons are related to personal factors. Furthermore, several model weaknesses were found: deficit in implementation of nonface-to-face model side, mainly lack of lecturers training, and low level of students training. However, the main conclusion is blended learning is a very powerful learning-teaching system in order to increase the educational level in latin american, although moving from traditional education to this systems has risks. 
KEYWORDS: blended-learning, higher education, educational demand, Latin America, demand.

\section{Introducción}

Los vertiginosos e inexorables cambios sociales, económicos, culturales, etc., que se vienen presentando en el mundo actual, están generando una serie de modificaciones en muchos aspectos que impactan en los escenarios nacionales, regionales y globales.

La educación, no está excluida de esa vorágine de movimientos dinámicos y sistemáticos. Como respuesta a las necesidades sociales surgen una diversidad de tendencias educativas: educación para adultos, educación a lo largo de la vida, educación continua, educación para todos, educación inclusiva, educación por competencias, etc.

Si nos referimos a la Educación Superior, en particular, encontramos una gran cantidad de modificaciones, que responden tanto a las demandas marcadas por los organismos internacionales y nacionales, como a la propia filosofía de cada una de las Institución de Educación Superior (IES), sin dejar de lado las características de los contextos donde ejercen su influencia educativa (UNESCO, 2008; Universidad de Guadalajara, 2014).

A continuación desarrollaremos una de las principales problemáticas que asumen las IES que es la atención a un número creciente de estudiantes, y la posibilidad de que a través de las TIC (Tecnologías de la Información y de la Comunicación) y con modelos semipresenciales se pueda atender a los mismos.

\subsection{El incremento de la demanda de Educación Superior en Latinoamérica}

En América Latina y el Caribe una proporción importante de personas que se les estratificaba como pobres han salido de esa categoría. En el informe del Banco Mundial elaborado por Ferreira et al. (2013) se afirma que el $43 \%$ de todos los habitantes de América Latina cambiaron de clase social, y que la mayor parte fue ascendente, y sólo el 2\% lo hizo de forma descendente. Este incremento en los datos se correlaciona con el gasto en salud pública y educación. Asimismo, se especifica que una parte importante del incremento de la clase media es de tipo emergente o vulnerable, que por sus condiciones de ingresos económicos se encuentran en el límite inferior de la clase media, y ello los coloca en riesgo de volver a la pobreza, en cuanto haya alguna pequeña crisis económica.

En América Latina se ha dado un avance de mejora de las condiciones económicas en las que viven los ciudadanos (a pesar de ser la Región del mundo con mayor desigualdad social) y ello ha propiciado el aumento de la demanda de servicios.

Aparejado a esta mejora económica y de calidad de vida, y fruto de la misma se ha producido una fuerte demanda de Educación Superior. Según los datos del informe del Centro Interuniversitario de Desarrollo (CINDA) y Universia (2011), de las Universidades Iberoamericanas, la matrícula de estudiantes inscritos en Instituciones de Educación Superior (IES) aumentó un 62\%, en el período 2000-2008, y de este porcentaje el 40,1\% corresponde al periodo 2004-2008. En el año 2008 la población de estudiantes en Iberoamérica ya había superado los 22 millones. El crecimiento anual de estudiantes en América Latina y el Caribe es del $6.8 \%$. Este alto crecimiento dificulta la incorporación de los mismos a las IES, y entre esas dificultades surge la de tener espacios físicos para atenderlos y profesorado cualificado. 
La UNESCO (2009) en la Conferencia Mundial sobre Educación Superior señala que no es suficiente con la incorporación de estudiantes a las IES, sino hacerlo con equidad y calidad.

La Universidad dentro de su responsabilidad social debe tener, entre otros, como objetivo medular, la calidad de la formación profesional que ofrece a sus alumnos. En el afán de incrementar la matrícula, se cae en trampas de las que a veces ya no es fácil salir, y que se están perpetuando cuando se masifica el ingreso de alumnos, y además, con mucha frecuencia, se diluye el rigor de la enseñanza-aprendizaje en las Universidades (Rojas, 2014).

En el informe Universia-CINDA (2011) se hace una fuerte crítica, a México, referente a la integración de estudiantes en las IES al afirmar que se ha preocupado especialmente en la tasa bruta de cobertura y no en crear escenarios favorables para la inclusión social.

Según la Asociación Nacional de Universidades de Instituciones de Educación Superior (ANUIES, 2013) el sistema de Educación Superior, en México, se componía de 3.309.221 estudiantes. La concentración de la matrícula en México se aglutina en algunos estados del centro, tales como el Estado de México y el Distrito Federal (mayormente concentrada en el segundo), seguidos de Veracruz y Jalisco. La evolución de la matrícula de la Educación Superior en México se ha sostenido de forma constante, habiéndose observado algunas dificultades, debido fundamentalmente a problemas de infraestructuras.

Algunas de las soluciones que se proponen, en México, es atender la matrícula a través de nuevas modalidades educativas, ya sea a través de las modalidades semipresenciales o apostarle a un proceso de virtualización, y en ambas modalidades basadas en una formación centrada en el alumno.

En esta línea la UNESCO recomienda, a los Estados Miembros, "apoyar una mayor integración de las TIC y fomentar el aprendizaje abierto y a distancia, con objeto de satisfacer el aumento de la demanda de Educación Superior" (UNESCO, 2009, p. 8).

Por último, García (2010), en la reunión convocada por el Instituto Internacional para la Educación Superior en América Latina y el Caribe, también expresa la importancia de las TIC como respuesta a la demanda de Educación Superior y para la internacionalización.

Las condiciones por las que se sugiere que las IES "Instituciones de Educación Superior" transiten hacia otra modalidad educativa tienen que ver con el crecimiento de la clase media, el aumento del bono demográfico y la dificultad de cobertura educativa que se observa trabajando bajo un modelo educativo tradicional.

\subsection{La educación semipresencial o blended-learning}

Actualmente nos encontramos con tres tipos de opciones: la educación presencial que ha sido la opción tradicional, la enseñanza a distancia y la educación semipresencial. Todas estas modalidades, no son otra cosa que, formas distintas de interacción entre el profesor-estudiante, metodologías diversas que se incorporan en el proceso enseñanza-aprendizaje, lugares donde se da el intercambio de ideas e información y conocimiento (aulas, espacios virtuales, PLE, comunidades virtuales de aprendizaje, etc.); frecuencia con la que se realizan las actividades; formas específicas de evidenciar el conocimiento; espacios donde se albergan y tiempos para el feeb-back hacia el alumno, etc.

En el ámbito educativo nos referiremos a la educación semipresencial como aquella que se caracteriza por una mezcla del cara a cara del alumno con el docente (educación presencial) y 
la utilización de la tecnología como complemento para sustituir esa otra parte del tiempo, en que el discente y el docente interactúan en una plataforma tecnológica o con distintas herramientas y/o aplicaciones que transmiten información y facilitan la comunicación.

Area, San Nicolás y Fariña, (2010), hacen una diferenciación de tres tipos de modelos de docencia, que están marcados o diferenciados de acuerdo al nivel o grado de presencialidad o distancia en la cual se dé la interacción entre profesor y alumno:

- Modelo de docencia presencial con Internet: el aula virtual como complemento o recurso de apoyo.

- Modelo de docencia semipresencial: el aula virtual como espacio combinado con aula física o blendedlearning.

- Modelo de docencia a distancia: el aula virtual como único espacio educativo.

Valenzuela (2006), nos da su definición de educación semipresencial: "El mismo término semipresencial nos indica que es un sistema o proceso educativo en el cual el estudiante no asiste diariamente a la clase" (p. 4). La autora, desglosa las características de la educación semipresencial, desde las características de cada uno de los agentes que se ven involucrados en ella: docente en el que destaca una predisposición a la innovación, a saber aplicar nuevas metodología y usar adecuadamente las TIC para promover la participación de los estudiantes; en los estudiantes resalta su mayor autonomía y responsabilidad, cambios en la forma de estudio y las posibilidades de interacción en encuentros presenciales y a través de las TIC; y respecto a los contenidos subraya un nuevo tratamiento de los mismos que deben posibilitar un trabajo más activo como cuestionar, analizar, sintetizar, etc. Resalta que además cobra una gran relevancia el diseño de los materiales educativos que se van usar.

La educación semipresencial no es fácil de implementar, en este sentido Llorente afirma:

El b-Learning es simple y complejo a la vez. Simple, porque se constituye básicamente como la combinación y/o integración de las experiencias del aprendizaje presencial con la experiencias del aprendizaje on-line; pero al mismo tiempo, resulta complejo si tenemos en cuenta que proporciona variadas posibilidades de implementación a través de un diseño virtual y presencial, y la multitud de contextos en los que puede ser aplicados. (Llorente, 2008, citada en Cabero; Llorente y Puente, 2010, p. 150).

Simón, Benedi y Blanché (2013) y sus colaboradores inciden en las dificultades de la implementación de la semipresencialidad que:

Integra los puntos fuertes de la presencialidad y de la virtualidad. Cuando el aprendizaje mixto está bien entendido y aplicado, la Educación Superior puede ofrecernos nuevos escenarios de reflexión crítica y creativa. El reto que se nos plantea es lograr una comprensión profunda de las necesidades, posibilidades y estrategias de aprendizaje en el marco de la semipresencialidad (p. 14).

Cada región o país tiene unas características peculiares y distintas a las de otros. Por tanto, para contextualizar un modelo académico semipresencial o virtual éste debe responder no sólo a la pertinencia que tiene para cada IES, sino que deben tomarse en cuenta los perfiles del profesorado (uno de los principales agentes dinamizadores en un cambio paradigmático de enseñanza-aprendizaje), el perfil del alumno, y su predisposición para cambiar de una formación tradicional a otra, en la que su participación es mucho más activa y crítica; los directivos que 
faciliten con recursos formativos, administrativos el desarrollo de esta modalidad; y sin menoscabar, desde luego, el rol del cambio pedagógico, metodológico y el tan vital acceso, uso y diversidad de las herramientas tecnológicas (Area, San Nicolás y Fariña, 2010; Cabero, Llorente y Morales, 2013; Turpo, 2010).

La capacidad de incorporar las TIC a la educación, no sólo da más posibilidades de acercar conocimientos a más lugares y personas salvando distancias; supone además una innovación en la educación. Al existir más posibilidades, el aprendizaje se ve modificado en comparación con una enseñanza más tradicional. Las prácticas educativas sufren una transformación, porque el uso de las TIC ofrece diferentes posibilidades que no pueden sino variar, en mayor o menor medida esta educación. Y en esta línea la educación semipresencial y la a distancia quedan reforzadas con el auge y fortalecimiento de las TIC.

La Educación mixta, blended-learning o semipresencial, es una modalidad que permite a las IES, el ingreso de más alumnos, pero debe suponer un cambio radical de formas de pensar, de actuar, de procesar y desde luego de aprender y enseñar. En esta modalidad educativa se ven involucrados los nuevos roles de los agentes y de los elementos de los procesos educativo: docentes, discentes, contenidos y metodologías, procesos administrativos, posturas de los directivos de las instituciones y desde luego el rol de la tecnología.

Por último, queremos centrarnos en el profesorado como una de las claves de la educación semipresencial, para ello vamos a acudir a Cabero et al. (2008, pp. 20-21) que enuncia claramente los roles que el profesor debe tener en estas nuevas modalidades donde se mezcla la presencialidad y la tecnología:

Rol del profesor:

- Presentación del a los estudiantes y de las normas de funcionamiento.

- Resolver de forma individual y colectiva las diferentes dudas que vayan surgiendo de interacción con los materiales que se le vayan presentando.

- Animar la participación de los estudiantes.

- Fomentar actividades de trabajo colaborativo y animar a la participación de todos los miembros.

- Realizar las valoraciones de las actividades realizadas. Desarrollar una evaluación continua formativa.

- Determinación de acciones individuales y grupales, en función de las necesidades de los diferentes estudiantes. Incitar a los alumnos para que amplíen y desarrollen sus argumentos propios y los de sus compañeros.

- Asesoramiento en métodos de estudio en la red. Facilitar y negociar compromisos cuando existan diferencias de desarrollo entre los miembros del equipo.

- Facilitar información adicional para la aclaración y profundización en conceptos.

- Ayudar a los alumnos en sus habilidades de comunicación señalándoles, en privado, sus posibles mejoras para un mayor entendimiento con el grupo, y seguimiento del proceso. 


\section{Método}

\subsection{Contexto de la investigación: el caso de la educación semipresencial en el Centro Universitario de los Valles, Universidad de Guadalajara, México}

El Centro Universitario de los Valles (CUValles) es uno (de los 15 centros) que conforman la red conocida como Universidad de Guadalajara. Él mismo se fundó en el año 2000, como consecuencia del proceso de descentralización que se inició en el año 1990, con objeto de huir del gigantismo y acercar los estudios universitarios a las distintas regiones del Estado de Jalisco (México).

El CUValles está ubicado en la Región 11 (Valles) del Estado de Jalisco, y recoge, fundamentalmente, a estudiantes de toda la Región. La región Valles tiene unos 350.000 habitantes distribuidos en poblaciones medianas y pequeñas, distantes entre ellas, con vías de comunicación que no están en buen estado y transportes públicos deficientes. Por ello, el desplazamiento al Centro Universitario es una dificultad importante para la gran mayoría de los estudiantes, por el elevado tiempo que invierten en llegar y también por el coste económico que supone.

Las condiciones expuestas, junto con el nivel bajo, de recursos económicos, de un alto porcentaje del alumnado, imposibilita a mucho estudiantes asistir todos los días. Es por ello, que el CUValles optó por aglutinar las clases, de las distintas licenciaturas y posgrados, en dos o tres días a la semana (considerando la mitad de la carga horaria, de cada materia o asignatura, para la presencialidad y la otra mitad para el trabajo virtual) y potenciar el uso de una plataforma Moodle para trabajar on-line con los estudiantes. Igualmente, abrió unos locales con computadoras y conexión a Internet, en las principales localidades, para facilitar la realización de las tareas a los estudiantes sin recursos.

La propuesta de una educación semipresencial fue, por tanto, una respuesta a una necesidad social, y además favoreció que el espacio (número de aulas) se pudiese optimizar abriendo más titulaciones. Es decir, era una buena solución, pero, ¿realmente lo es?

\subsection{Tipo de investigación, instrumentos, universo y muestra}

En el CUValles se planificó una evaluación institucional, realizándose una investigación exploratoria sobre la implementación del modelo semipresencial, que se centró en el profesorado y en los estudiantes.

Con el profesorado se utilizó la herramienta de focusgroup con profesores de cada departamento y con los estudiantes se utilizó una metodología tipo encuesta. Para acercarnos a las vivencias y motivaciones, del alumnado que está estudiando en CUValles, hicimos un cuestionario ad-hoc, con 53 variables. El cuestionario fue cumplimentado en línea con la aplicación lime-survey, y las preguntas fueron de tipo escala Likert con opciones de respuestas impares.

El cuestionario tuvo 53 ítems sobre aspectos como la valoración de: la docencia y tipos de actividades que se realizaban; del uso de la plataforma Moodle y las herramientas TIC alojadas en la misma; los servicios complementarios que ofrece el centro educativo (biblioteca, gimnasio,...); el modelo semipresencial; y la formación integral complementaria que se ofrece en seminarios y talleres voluntarios. Es por ello, que en este artículo sólo nos vamos a centrar en la percepción y valoración del modelo semipresencial. 
El cuestionario fue cumplimentado por 1.755 alumnos (muestra), que estudian en las once licenciaturas que ofrece el CUValles, y suponen casi el $60 \%$ de los estudiantes de la población, de licenciatura, que estaban matriculados en el curso 2014A.

El tratamiento de los datos incluyó el cálculo de medias, el estudio de correlaciones entre variables y la distribución porcentual de las respuestas. Se dieron correlaciones en algunas preguntas o ítems, en función del sexo y las licenciaturas que estudiaban los encuestados, pero que no fueron significativas. En las variables que se muestra en este artículo hemos incluido sólo la distribución de porcentajes de las respuestas para no sobresaturar de información, y por estimar que es la que mejor visualiza las opiniones de los estudiantes.

\section{Resultados}

En los focusgroup la información clave que aportaron los profesores, de manera generalizada, se concrentán en las siguientes afirmaciones:

- Los profesores noveles (con menos de dos años) se encuentran con dudas y carencias formativas importantes, para atender al alumnado en esta modalidad. Sus debilidades la encuentran en la carencia de formación pedagógica y en la incertidumbre que les genera diversificar la docencia en forma presencial y virtual.

- Los profesores, en general, han recibido cursos (en los periodos sin actividad académica) sobre el modelo semipresencial, pero sienten que los mismos no les han servido para mejorar de forma significativa su planificación didáctica, ni su trabajo como docente.

- El profesorado valora que el nivel general de sus estudiantes es bajo o muy bajo, y que llegan estudiantes con deficiencias formativas básicas en Matemática y Lengua (lectura comprensiva).

- El profesorado se queja de la poca predisposición de los estudiantes para realizar las tareas colocadas en la plataforma.

- Parte importante, del profesorado, está convencido de que el modelo semipresencial conlleva un menor aprendizaje de los estudiantes, en comparación con el modelo presencial, debido a que tienen menos horas presenciales con los estudiantes. Está opinión está más arraigada en los profesores que imparten clases en las licenciaturas de ingenierías.

- El profesorado ha recibido formación para el uso de la plataforma Moodle, pero reconoce que tienen carencias educativas para optimizar su uso.

Esta información clave fue complementada con los datos obtenidos por un cuestionario. En todos los programas educativos cumplimentaron, el cuestionario, más del $50 \%$ de los estudiantes que componen la población.

La muestra de estudiantes de CUValles se ha distribuido entre un 56.9\% de mujeres y un $43.1 \%$ de hombres, porcentaje muy similar al de la población. No obstante, la distribución de género presenta importantes diferencias en función de las licenciaturas. Así tenemos algunas con una fuerte presencia de mujeres como Trabajo Social con el 92.5\%, Psicología con el $81.7 \%$ y Educación con el 78.6\%; y otras con una gran concentración de hombres como Mecatrónica con el 93.4\%, Electrónica y Computación con el 78.3\% y Agronegocios con el 78\%. 
El 60.6\%, de los estudiantes, de la muestra de CUValles, está de acuerdo con el modelo semipresencial, el 26.6\% (más de una cuarta parte de los estudiantes) está parcialmente de acuerdo, y el $12.8 \%$ (una octava parte) está en desacuerdo con el modelo.

Los menores porcentajes "de acuerdo" corresponde a los estudiantes de Contaduría con el 44.5\%, Psicología con el 48.9\% y Mecatrónica con el 49.6\%; y los mayores los tienen los alumnos de las licenciaturas de Educación con un 79.2\%, Agronegocios con el 71.2\% y Trabajo Social con el $70.8 \%$. Estos datos evidencian que hay una gran diferencia en las licenciaturas, el $34.8 \%$ entre el mayor y el menor porcentaje, en el acuerdo que suscita el modelo semipresencial.

Igualmente, los datos reflejan que las tres carreras tecnológicas Mecatrónica, Sistemas y Electrónica y Computación tienen unos porcentajes "de acuerdo" por debajo de la media.

También resaltamos que si se suman los porcentajes de "parcialmente de acuerdo" y en "desacuerdo" el porcentaje es del $39.4 \%$, evidenciando que para un alto porcentaje del total de estudiantes, y en dos licenciaturas para la mayoría, el modelo semipresencial es cuestionado. licenciatura.

En la gráfica se muestran los porcentajes de la respuesta "de acuerdo", de cada

\section{Gráfica 1. De acuerdo con el modelo presencial}

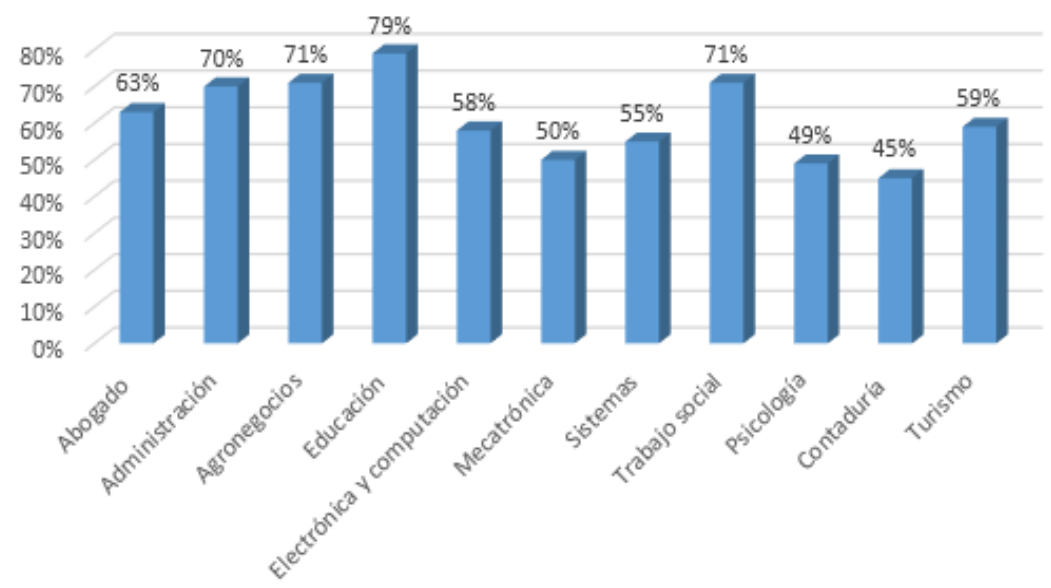

Fuente: elaboración propia

Las respuestas de los estudiantes nos indican que el $54.5 \%$, de la muestra de CUValles, afirma estar de acuerdo con el modelo semipresencial porque le supone un ahorro de tiempo, pero para un $45.5 \%$ el ahorro de tiempo no supone un motivo para apoyar al modelo.

Las licenciaturas con mayores porcentajes, de estudiantes, que indican que están de acuerdo con la semipresencialidad por el ahorro de tiempo son Educación con el 66.9\%, Mecatrónica con el 62\%, Electrónica con el 60.4\%; y las que tienen los menores porcentajes son Psicología con el $38.9 \%$, Sistemas con el $42.6 \%$ y Trabajo Social con el $50.8 \%$.

Las diferencias entre las licenciaturas son relevantes, entre el menor porcentaje de acuerdo de Psicología y el mayor de Educación, el 28\%.

En la gráfica siguiente se muestran los porcentajes de la respuesta "de acuerdo" de los estudiantes de las licenciaturas. 
Gráfica 2. De acuerdo, con el modelo semipresencial, por ahorro de dinero

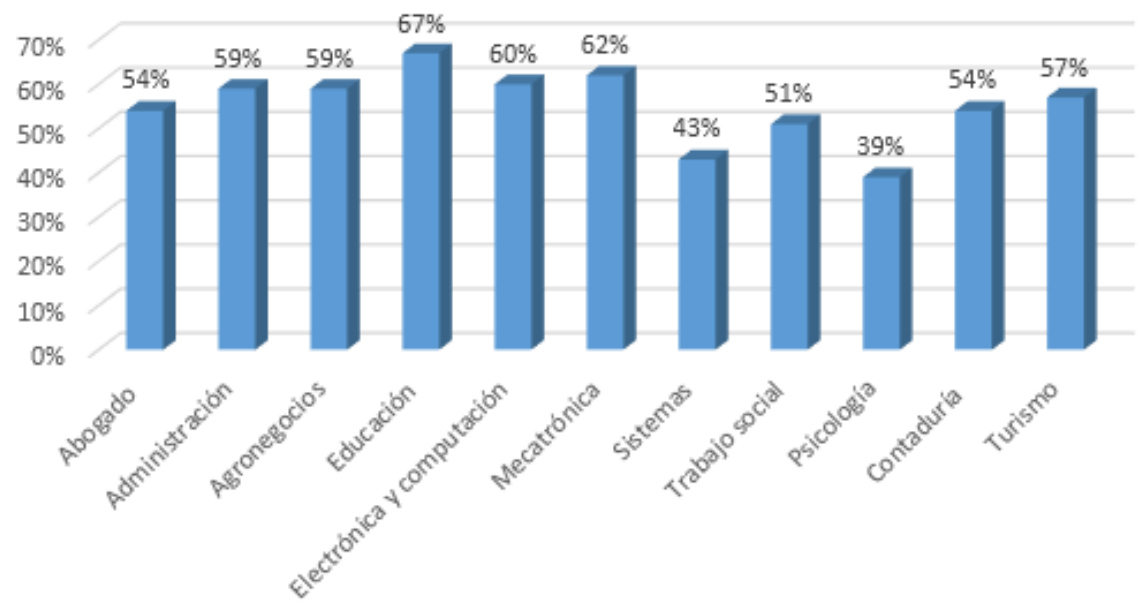

Fuente: elaboración propia

El motivo que tiene el mayor porcentaje de acuerdo, para apoyar el modelo semipresencial, entre los estudiantes es el ahorro de dinero, ya que el $66.7 \%$ lo afirman. Es decir, para dos tercios del alumnado éste es un motivo, pero no lo es para el otro tercio (el 33.3\%).

Los mayores porcentajes de estudiantes que están de acuerdo, por el ahorro de dinero, lo tienen Educación con el 82.5\%, Agronegocios con el 75\% y Administración con el 72.6\%; y los menores porcentajes corresponden a los de Psicología con el 59.4\%, Mecatrónica con el 59.5\% y Turismo con el $60.3 \%$. Ello supone una diferencia del $23.1 \%$, que es importante, entre el mayor y el menor porcentaje de respuestas "de acuerdo". En el gráfico, se reflejan los porcentajes de las respuestas "de acuerdo".

Gráfica 3. Acuerdo, con el modelo semipresencial, por ahorro de dinero

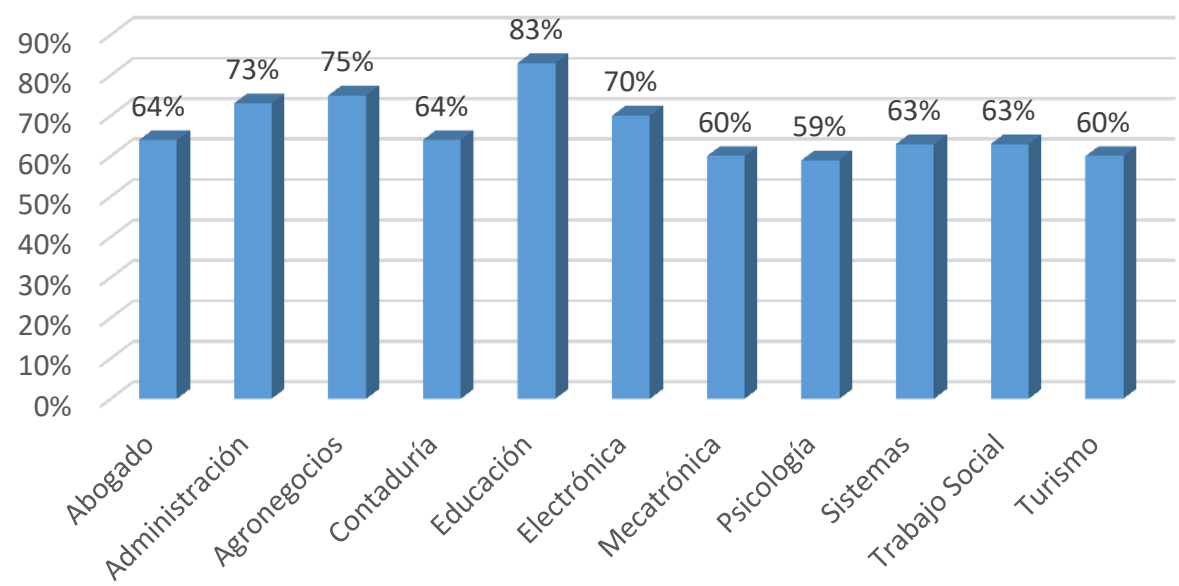

Fuente: elaboración propia 
Los motivos familiares son, para el $14.7 \%$ de los estudiantes de CUValles, una causa para estar "de acuerdo" con el modelo de CUValles. Por otro lado, para el 85.3\% de los estudiantes este motivo no es suficiente para estar de acuerdo.

Los mayores porcentajes de apoyo, por esta causa, corresponden a los estudiantes de las licenciaturas de Trabajo Social con el 20.8\%, Educación con el 19.8\% y Psicología con el 17\%; y los menores los tienen los de Mecatrónica con el 6.6\%, Electrónica con el 8.5\% y Administración con el $12.4 \%$. La diferencia entre el mayor y el menor porcentaje, "de acuerdo" por causas familiares, de las licenciaturas es del $14.2 \%$.

En el Centro Universitario hay un porcentaje alto de estudiantes, el $45.8 \%$, que aluden al trabajo como un elemento que le hace estar de acuerdo con el modelo semipresencial, pero no es una causa para el 54.2 de los estudiantes, es decir, para más de la mitad del alumnado.

Los mayores porcentajes "de acuerdo" por causas laborales se dan en las licenciaturas de Agronegocios con un 59.1\% (el sector agrario es muy relevante en la Región y muchos estudiantes de Agronegocios trabajan en él), Turismo con un 51.9\% y Abogado con el 50.6\%; y los menores porcentajes lo tienen los estudiantes de Psicología con el 29.7\%, Electrónica con el $40.6 \%$ y Sistemas con el $40.7 \%$.

No se sabe exactamente, si el modelo semipresencial empuja a los estudiantes a buscar un empleo para optimizar su tiempo libre, o bien las condiciones económicas de las familias y de los estudiantes empujan a los mismos a trabajar para contribuir al mantenimiento económico. Es evidente que una mayor disposición de tiempo favorece la empleabilidad. Los porcentajes de estudiantes de las licenciaturas que están “de acuerdo" por motivos laborales están expresados en la siguiente gráfica.

Gráfica 4. Acuerdo, con el modelo semipresencial, por causas laborales

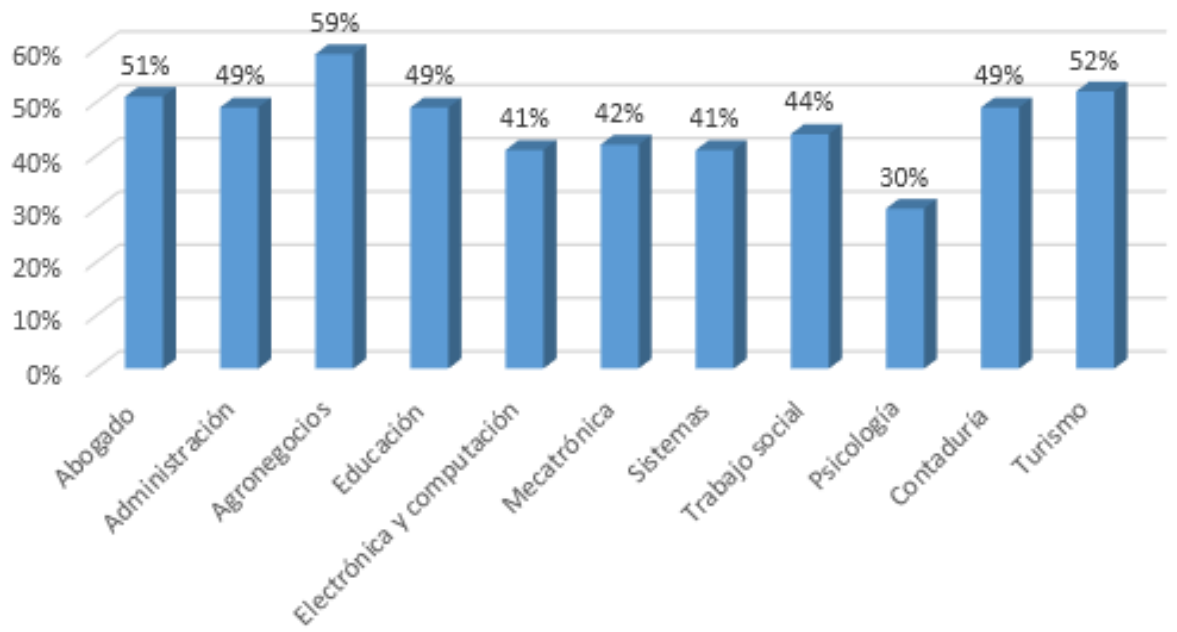

Fuente: elaboración propia

En los modelos semipresenciales la retroalimentación de las tareas tienen un mayor protagonismo, para orientar a los estudiantes en su proceso de aprendizaje. Es por ello que preguntamos si los profesores daban comentarios sobre las tareas. 
Los estudiantes tenían cinco opciones y la respuesta más seleccionada fue la neutra "algunos" profesores, que fue elegida por el $43.8 \%$ de los estudiantes. Las opciones que indican una valoración positiva son "casi todos los profesores", que es elegida por el $28.9 \%$ de los estudiantes, y "todos los profesores" seleccionada por el $11.1 \%$. La suma de las valoraciones positivas llega al 40\%, que es un porcentaje menor (un 3.8\%) que el de la valoración neutra. Las valoraciones negativas, del nivel de retroalimentación del profesorado, "casi ninguno" es respondida por el $13.8 \%$ y la de "ninguno" un $2 \%$, la suma de ambas valoraciones alcanza el $15.8 \%$.

Los datos señalados nos muestran que una sexta parte de los estudiantes hacen una valoración negativa del profesorado, sobre la retroalimentación de sus tareas. Cuatro de cada diez estudiantes, hace una valoración positiva y el mayor porcentaje lo tiene la opción neutra "algunos profesores".

Las licenciaturas que tienen los mayores porcentajes, de respuestas positivas (casi todos o todos de los profesores) son: Administración con el 52.7\%, Contaduría con el $46 \%$ y Abogado con el 44.7\%; y las que tienen los menores porcentajes de retroalimentación son los de las licenciaturas de Electrónica con el 25.5\%, Educación con el 26.6\% y Mecatrónica con el 33\%. La diferencia entre el mayor porcentaje de Administración y el menor de Electrónica es el 27.2\%, que es una diferencia importante.

En la siguiente gráfica se muestran los porcentajes de valoraciones positivas.

Gráfica 5. Retroalimentación de las tareas

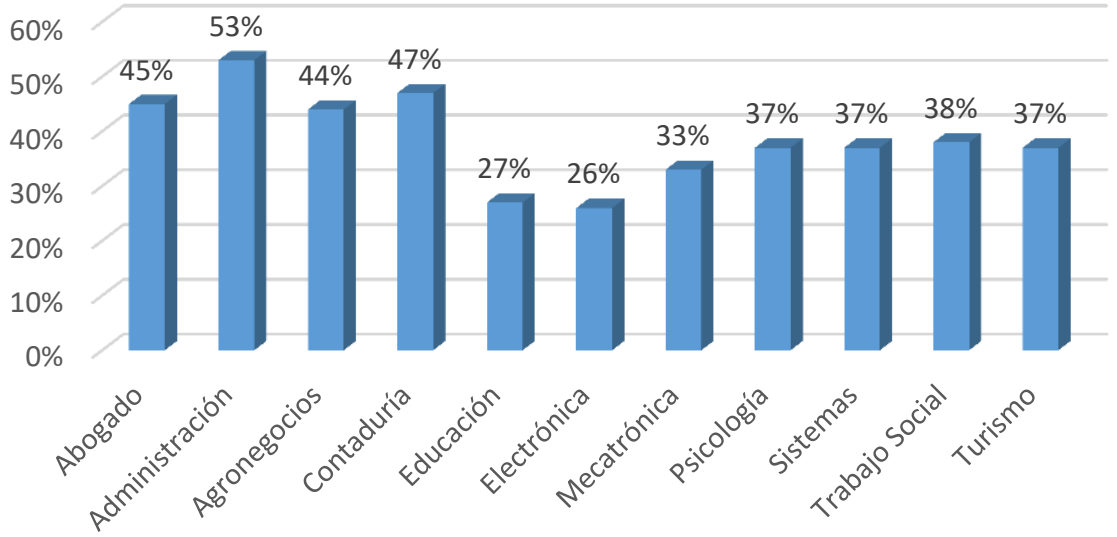

Fuente: elaboración propia

El 24.3\% de los estudiantes, de la muestra de CUValles, creen que las actividades en línea sustituyen la presencialidad. Este porcentaje evidencia una falta de confianza en las actividades en línea, de cara a compensar la presencialidad.

Es muy importante resaltar que más del $75 \%$ de los estudiantes no creen que las actividades en línea compensan que haya menor presencialidad, lo que nos lleva a inferir que asumen el modelo semipresencial por los motivos personales que se han señalado en las preguntas anteriores, siendo conscientes de que tienen un perjuicio, en su aprendizaje, por el funcionamiento de las actividades en línea, y la anteriormente señalada falta de retroalimentación de parte del profesorado. 
Comparando las distintas titulaciones los menores porcentajes "de acuerdo", con el modelo semipresencial, corresponden a las licenciaturas de Psicología con el 14\%, Turismo con el $18.3 \%$ y Trabajo Social con el 20\%; y los mayores porcentajes los tienen las licenciaturas de Administración con un 35\%, Educación con el 31\% y Psicología con el 17\%. La diferencia entre el mayor porcentaje, de Administración, y el menor porcentaje de Psicología es del 21\%, entre ambas licenciaturas.

Los datos resaltan una gran debilidad del modelo semipresencial, puesto que sólo un tercio de los estudiantes de Administración (que tiene el mayor porcentaje "de acuerdo") creen que las actividades en línea sustituyen la presencialidad.

\section{Gráfica 6. Las actividades en línea sustituyen la presencialidad}

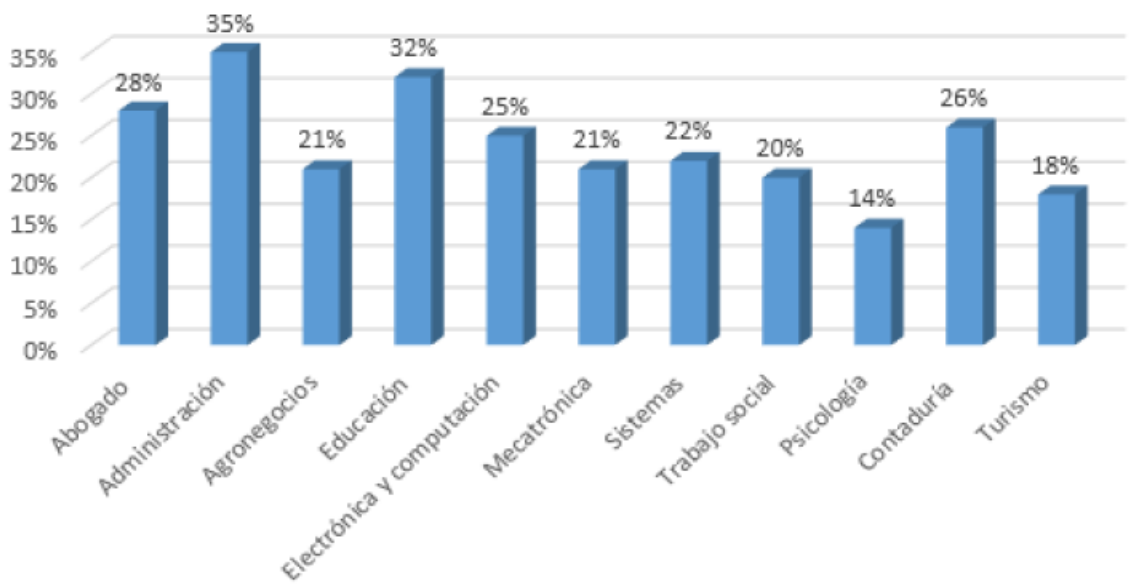

Fuente: elaboración propia

\section{Conclusiones o discusión}

Las Universidades Latinoamericanas y el Caribe sufren una fuerte presión para atender a un número creciente de estudiantes. Los porcentajes de crecimiento elevan la dificultad, tanto de los gobiernos de los países como de las universidades públicas y privadas, para responder a la demanda y hacerlo con calidad.

La educación a distancia y la semipresencial, ambas mediadas por TIC, disminuyen una de las principales dificultades que es la falta de espacios adecuados para la docencia y pueden ofrecer una alternativa a la educación presencial. La semipresencialidad mejora la optimización de los espacios, ya que pueden absorber a muchos más estudiantes (aproximadamente un $40 \%$ más) facilitando poder responder a la demanda social de formación universitaria.

En el caso del CUValles, todos los espacios están ocupados durante toda la semana, por lo que si se pasase a un sistema presencial como mínimo se debería reducir el número de estudiantes en una tercera parte. 
La semipresencialidad aporta grandes ventajas para las ciudades, con una alta concentración de la población, en Iberoamérica, que es donde suelen estar ubicadas las Universidades, ya que favorece la movilidad. En una universidad, como la de Universidad de Guadalajara, con una población de más de 120.000 estudiantes de enseñanza superior, un modelo semipresencial que conllevara el desplazamiento de los estudiantes tres días a la semana supondría un ahorro del $40 \%$ de la movilidad, es decir, que se ahorrarían unos 48.000 desplazamientos diarios, con una mejora indudable de la circulación de vehículos, un ahorro energético, de desgastes de automóviles, una disminución de la contaminación atmosférica, las áreas metropolitanas serían más sustentables y mejoraría la calidad de toda la población.

El ahorro de tiempo suele ser una de las razones más señaladas, en todos los estudios y planteamientos teóricos, del beneficio de la semipresencialidad. El tiempo invertido en los desplazamientos de ida y vuelta a los campus suele ser relevante en la jornada de los estudiantes. Tiempo que difícilmente puede ser útil, que genera cansancio y en muchas ocasiones produce un nivel alto de estrés a los estudiantes, puesto que cuando los desplazamientos son largos están sometidos a muchas variables relacionadas con el transporte seleccionado.

Es evidente que asistir menos días, al Centro Universitario, supone un ahorro de dinero, pero para un tercio de los estudiantes, de CUValles, el ahorro de dinero no es una causa para apostar por la semipresencialidad. Esto puede ser debido a dos causas: una que hay alumnos que tienen solvencia económica y el ahorro no es para ellos una motivación, pero otra puede ser que, aunque no tengan una situación holgada, prefieran invertir dinero en asistir al centro más días, porque crean que ello les trae un beneficio mayor que la inversión económica que hacen para ir al Centro.

La reducción del tiempo y de días de asistencia, a los Centros Universitarios, supone un ahorro de tiempo y mejora la calidad de vida de los estudiantes. Pero, ¿la calidad del servicio recibido en las horas presenciales, las actividades virtuales y el seguimiento de las mismas propician un aprendizaje de la misma calidad que cuando se tiene más tiempo de contacto con el profesorado?; ¿el tiempo de ahorro de la movilidad al centro universitarios compensa?, es decir, ¿la semipresencialidad ahorra efectivamente tiempo?, o ¿intercambia tiempo de transporte por un mayor tiempo de aprendizaje en casa para llegar al mismo nivel de aprendizaje?

Los alumnos, que han participado en la investigación realizada en el CUValles, creen que las actividades que realizan no sustituyen la menor presencialidad. Es decir, que estiman que aprenderían más con un sistema presencial, esta percepción de los estudiantes es muy relevante.

Las respuestas de los estudiantes señalan un problema importante, la retroalimentación de las tareas es siempre importante en cualquier tipo de modelo, porque mejora el aprendizaje de los estudiantes. En un sistema semipresencial la importancia de la retroalimentación es mayor, debido a que parte del tiempo de trabajo de los estudiantes se realiza a través de tareas, sin la presencia física del profesor, por lo que si ese trabajo no es retroalimentado se generarán incertidumbres importantes en los estudiantes. Es por ello, que la respuesta idónea sería la opción "todos los profesores" retroalimentan las tareas, que debería ser el 100\% de las respuestas de los estudiantes, pero que sólo es elegida por el $11.1 \%$ de los estudiantes, evidenciando una fuerte crítica, que puede llegar a poner en duda que el sistema sea efectivamente semipresencial.

Si en un Centro Universitario como es CUValles, en el que se ha introducido de forma pertinente la educación semipresencial, para solucionar un problema de su contexto social, que cuenta con un profesorado que de forma mayoritaria ha integrado en sus prácticas docentes 
metodologías como la de elaboración de proyectos y el trabajo colaborativo de los estudiantes, tienen una percepción de que su nivel de aprendizaje sería superior con una mayor presencialidad, ¿hay que preguntarse por qué?

En función de las respuestas de los estudiantes de CUValles el punto débil está en las tareas virtuales y en la retroalimentación de las mismas, ¿cuál puede ser la causa?

Los profesores que se incorporan al CUValles saben trabajar en la educación presencial, pero no en la virtual. La educación semipresencial exige un dominio del profesorado de dos formas de trabajar, la presencial y la a distancia o virtual, en la que la rápida respuesta a los estudiantes es una de las claves del éxito y evita que haya elevadas tasas de abandono. Las opiniones del profesorado manifestando su sensación de no estar capacitados pedagógicamente para este modelo, a pesar de que muchos han recibido formación en cursos ofrecidos por el centro, evidencian la dificultad de implementar el modelo semipresencial y la gran complejidad que refleja la cita de Llorente (2008) y la de Simón et al. (2014) que hemos introducido en la parte teórica.

La escasa retroalimentación, de las tareas, hace que los estudiantes de CUValles se sientan inseguros y desatendidos y es por ello, que a pesar de las evidentes ventajas, de la educación semipresencial que reciben, la gran mayoría de los estudiantes creen que con la educación presencial sería mejor para su aprendizaje. Estas afirmaciones coinciden con los temores de una disminución de la calidad que se reflejaba en Rojas (2014).

Si acudimos de nuevo a Valenzuela (2006) y Cabero et al. (2008) apreciamos que hay una serie de deficiencias que impiden el afianzamiento del modelo semipresencial, en el CUValles, con calidad. Las principales se concentran en los estudiantes, que según los profesores no son lo suficientemente participativos y muchos de ellos tienen un bajo nivel formativo; y por otro lado está la falta de retroalimentación de las tareas por parte del profesorado.

Si las Instituciones de Educación Superior promueven la educación semipresencial sin una formación del profesorado, adecuada para esta modalidad, y sin un control de que algunas prácticas docentes son distintas en la enseñanza semipresencial, es muy probable que esa sensación y quizás realidad de un menor aprendizaje (que con la enseñanza presencial) se extienda entre los estudiantes.

La existencia de plataformas institucionales como puede ser la Moodle no garantiza una transición de un modelo presencial hacia la semipresencialidad, ya que éstas son también usadas como herramientas en los modelos presenciales.

Hasta el momento la educación había sido presencial, o bien a distancia (virtual), el avance hacia la semipresencialidad se ha dado fundamentalmente desde la educación a distancia incluyendo algunas sesiones de trabajo presencial con los estudiantes (que suponen un bajo porcentaje del total de horas). Un cambio hacia una educación semipresencial en la que los porcentajes de presencialidad y virtualidad se repartan de forma más equilibrados, como es el caso del CUValles con un 50\% para cada modalidad conlleva unos cambios importantes. Es necesario profundizar en investigaciones educativas sobre metodologías, formas de atender a los estudiantes, etc., en la educación semipresencial.

La educación semipresencial aporta grandes ventajas personales para los estudiantes, y como hemos señalado puede ser una solución para atender el incremento de la demanda de Educación Superior, pero ella sólo podrá funcionar con calidad si el profesorado sabe trabajar de 
dos formas distintas: presencial y virtual.

Simon, Benedí y Blanché (2013) en su investigación sobre la semipresencialidad en las Universidades de la Xarxa Vives, inciden en la importancia de la formación del profesorado para la semipresencialidad, ya que ésta exige nuevas competencias que agrupan en tres ámbitos: competencias cognitivas, competencias comunicativas y competencias afectivas. Del listado de competencias, que ellos proponen, destacamos entre las cognitivas las de "instruido en la gestión documental y la gestión avanzada de la información y conocedor de los métodos didácticos para entornos presenciales y virtuales"; y de las comunicativas la "perseverante en la retroalimentación del trabajo del estudiante" (p. 90). Igualmente, en su estudio proponen un cambio de roles en el estudiante, sobre este aspecto la opinión expresada por el profesorado de CUValles sobre la poca predisposición de los estudiantes para realizar las tareas colocadas en la plataforma Moodle, supone también una grave dificultad para el éxito del sistema semipresencial.

Concluimos que la formación del profesorado es clave para la implementación de modelos semipresenciales. Avanzar en planificaciones que implementen estos modelos, con calidad, pueden ser un tema prioritario en la Educación Superior en Iberoamérica. Igualmente, las IES no pueden creer que va a generar una reducción de profesorado, ni de horarios de dedicación del profesorado, puesto que la retroalimentación a las tareas del alumno y el contacto, mediado por TIC, es importante para el éxito de la educación semipresencial, y ello conlleva una alta dedicación de tiempo.

La mejor atención a la familia, el ahorro económico y la posibilidad de poder acceder al mundo laboral (a tiempo parcial) son grandes beneficios que aportan una educación semipresencial y son unas buenas razones para apostar por ella, pero no pagando el precio de una reducción de la calidad de enseñanza que reciben los estudiantes.

\section{Referencias bibliográficas}

ANUIES (2013). Anuarios estadísticos de Educación Superior. Recuperado de: http://www.anuies.mx/iinformacion-y-servicios/informacion-estadistica-de-educacionsuperior/anuario-estadistico-de-educacion-superior

Área, M.; San Nicolás, M. B. y Fariña, E. (2010). Buenas Prácticas de aulas virtuales en la docencia Universitaria Semipresencial. Teoría de la Educación. Educación y Cultura en la Sociedad de la Información, 1 (11), 7-31. Recuperado de : http://revistatesi.usal.es/ revistas_trabajo/index.php/revistatesi/article/view/5787/5817

CINDA y UNIVERSIA (2011). Educación Superior en Iberoamérica. Informe 2011. Santiago de Chile. Recuperado de: http://www.cinda.cl/wp-content/uploads/2014/02/EducaciónSuperior-en-Iberoamérica-2011.pdf

Cabero, J.; Ballesteros, C.; Barroso, J.; Llorente, M. C.; Morales, J. A. ; Romero, R. y Román, P. (2008). Aportaciones al e-learning: desde la investigación educativa. Sevilla: Grupo de Investigación Didáctica, Universidad de Sevilla. Recuperado de: https://www.researchgate.net/publication/234112206_APORTACIONES_AL_ELEARNING_DESDE_LA_INVESTIGACION_EDUCATIVA 
Cabero, J.; Llorente, M. C. y Morales, J. A. (2013). Aportaciones al e-learning desde un estudio de buenas prácticas en las universidades Andaluzas. Revista de Universidad y de Sociedad del Conocimiento, 1 (10), 45-58, doi: http://dx.doi.org/10.7238/rusc.v10i1.1159

Cabero, J.; Llorente, M. C. y Puentes, Á. (2010). La satisfacción de los estudiantes en red en la formación semipresencial. Revista Científica de Comunicación y Educación. Comunicar, 35 (18), 149-157, doi: 10.3916/C35-2010-03-08

Ferreira, F. H. et al (2013). La movilidad económica y el crecimiento de la clase media en América Latina. Washington, D.C.: Banco Mundial. Recuperado de: https://openknowledge.worldbank.org/bitstream/handle/10986/11858/9780821397527.pdf

García, C. (2010). Construcción de un espacio de Educación Superior Latinoamericano. En Cadenas, J.M. (coord.). La Universidad latinoamericana en discusión. Caracas: UNESCO. Recuperado de; http://portal.unesco.org/geography/es/ev.phpURL_ID=13702\&URL_DO=DO_TOPIC\&URL_SECTION=201.html

Rojas, J. (2014). Construyamos hoy la universidad del futuro. Propuesta para una nueva gestión universitaria en un mundo global. Concepción (Chile): Universidad de Concepción. Recuperado de: http://www2.udec.cl/ jrojas/Construyamos_hoy.pdf

Simón, J.; Benedí, C. y Blanché, C. (2013). La semipresencialidad como respuesta a los nuevos retos de la universidad. Barcelona: Octaedro y Universidad de Barcelona.

Turpo, O. W. (2010). Contexto y desarrollo de la modalidad Educativa Blended-Learning en el Sistema Universitario Iberoamericano. Revista Mexicana de Investigación Educativa, 45 (15), 345-369. Recuperado de: http://www.redalyc.org/pdf/140/14012507002.pdf

Universidad de Guadalajara (2014). Plan de Desarrollo Institucional 2014-2030. Construyendo el futuro.Guadalajara: Universidad de Guadalajara. Recuperado de http://www.copladi.udg.mx/content/pdi-2014-2030

UNESCO (2009). Conferencia Mundial de Educación Superior: La nueva dinámica de la Educación Superior y la Investigación para el cambio social y el desarrollo. Paris: UNESCO. Recuperado de:

http://www.unesco.org/education/WCHE2009/comunicado_es.pdf

Valenzuela, A. E. (2006). Apuntes para una Educación Semipresencial. Guatemala: Universidad Rafael Landívar. Recuperado de: http://biblio3.url.edu.gt/PROFASR/ModuloFormacion/07.pdf 